\title{
THURSTON'S SPINNING CONSTRUCTION AND SOLUTIONS TO THE HYPERBOLIC GLUING EQUATIONS FOR CLOSED HYPERBOLIC 3-MANIFOLDS
}

\author{
FENG LUO, STEPHAN TILLMANN, AND TIAN YANG \\ (Communicated by Daniel Ruberman)
}

\begin{abstract}
We show that the hyperbolic structure on a closed, orientable, hyperbolic 3-manifold can be constructed from a solution to the hyperbolic gluing equations using any triangulation with essential edges. The key ingredients in the proof are Thurston's spinning construction and a volume rigidity result attributed by Dunfield to Thurston, Gromov and Goldman. As an application, we show that this gives a new algorithm to detect hyperbolic structures and small Seifert fibred structures on closed 3-manifolds.
\end{abstract}

\section{INTRODUCTION}

In his Princeton notes [29, Thurston introduced a system of algebraic equations (called the hyperbolic gluing equations) to construct hyperbolic metrics on orientable 3-manifolds with torus cusps. He used solutions to the hyperbolic gluing equations to produce a complete hyperbolic metric on the figure-eight knot complement in the early stages of formulating his geometrization conjecture. On a closed, triangulated, oriented 3-manifold $M$, the hyperbolic gluing equations can be defined in the same way: We assign to each edge of each oriented tetrahedron in the triangulation a shape parameter $z \in \mathbb{C} \backslash\{0,1\}$, such that

(a) opposite edges of each tetrahedron have the same shape parameter;

(b) the three shape parameters assigned to the three pairs of opposite edges in each tetrahedron are $z, \frac{1}{1-z}$ and $\frac{z-1}{z}$ subject to an orientation convention; and

(c) for each edge $e$ in $M$, if $z_{1}, \ldots, z_{k}$ are the shape parameters assigned to the tetrahedron edges identified with $e$, then we have

$$
\prod_{i=1}^{k} z_{i}=1 .
$$

The equations (11) are termed the hyperbolic gluing equations, and the set of all solutions is the parameter space $\mathcal{P}(M)$. The space $\mathcal{P}(M)$ depends on the triangulation of $M$. We will study the solutions using a volume function and representations

Received by the editors October 2, 2010 and, in revised form, April 8, 2011.

2010 Mathematics Subject Classification. Primary 57M25, 57N10.

Key words and phrases. Hyperbolic 3-manifold, triangulation, parameter space, Thurston's gluing equations.

Research of the first and third authors was supported in part by the NSF.

Research of the second author was partially funded by a UQ New Staff Research Start-Up Fund and under the Australian Research Council's Discovery funding scheme (DP1095760). 
of the fundamental group. In order to produce the representations, we need the following topological hypothesis.

The edge $e$ in $M$ is termed essential if it is not a null-homotopic loop in $M$. This is clearly the case if it has distinct end-points, but we allow the triangulation of $M$ to be semi-simplicial (or singular), so that some or all edges may be loops in $M$. We will always assume that all edges are essential. In this case, to each solution $Z \in \mathcal{P}(M)$, there is an associated representation $\rho_{Z}: \pi_{1}(M) \rightarrow P S L_{2}(\mathbb{C})$. Given $Z \in \mathcal{P}(M)$, the volume of $Z$, denoted $\operatorname{Vol}(Z)$, is defined using the LobachevskyMilnor formula [19] as the sum of the signed volumes of the oriented ideal hyperbolic tetrahedra specified by $Z$.

Suppose that $M$ is hyperbolic and denote by $\operatorname{Vol}(M)$ the hyperbolic volume of $M$. Using results about representation volume, we show that for each $Z \in \mathcal{P}(M)$, $\operatorname{Vol}(Z) \in[-\operatorname{Vol}(M), \operatorname{Vol}(M)]$, and it is a consequence of the Schläfli formula that Vol: $\mathcal{P}(M) \rightarrow \mathbb{R}$ is constant on the topological components of $\mathcal{P}(M)$. Our main observation is that, just as in the case of hyperbolic manifolds with cusps, we can recover the hyperbolic structure on a closed hyperbolic 3-manifold from any solution of the hyperbolic gluing equations with maximal volume:

Theorem 1.1. Let $M$ be a closed, oriented, triangulated, hyperbolic 3-manifold with the property that all edges in $M$ are essential. Then there exists $Z_{\infty} \in \mathcal{P}(M)$ such that $\operatorname{Vol}\left(Z_{\infty}\right)=\operatorname{Vol}(M)$. Moreover, for any such $Z_{\infty}$, the associated holonomy representation $\rho_{\infty}: \pi_{1}(M) \rightarrow P S L_{2}(\mathbb{C})$ is discrete and faithful, and $M$ is isometric with $\mathbb{H}^{3} / \rho_{\infty}\left(\pi_{1}(M)\right)$.

The existence statement in the proof of Theorem 1.1 is constructive and makes crucial use of Thurston's spinning construction from [30]. The remainder is proved using a volume rigidity result attributed by Dunfield to Thurston, Gromov and Goldman (see [7, Theorem 6.1).

The only known algorithmic detection and description of hyperbolic structures on closed 3-manifolds is due to Casson and Manning [17] and uses the automatic structure of the fundamental group. As an application, we show that the above results not only allow the algorithmic construction of the hyperbolic structure without having to find a solution to the word problem, but that they reduce this construction to algorithms from the mainstream in computational algebraic geometry. Moreover, we show that the recognition of small Seifert fibred spaces is also within the scope of our approach:

Theorem 1.2 (Casson-Manning [17] and Rubinstein [27]). There exists an algorithm, which will, given a triangulated, closed, orientable 3-manifold $M$ decide whether or not it has a hyperbolic structure or a small Seifert fibred structure. Moreover, if $M$ has a hyperbolic structure or a small Seifert fibred structure, then the structure is algorithmically constructible.

This paper is organised as follows. In Section 2, we summarise some background material on hyperbolic geometry and prove a technical lemma which will be used in the spinning construction. Thurston's spinning construction is reviewed in Section 3, and basic properties of the parameter space are given in Section 4 The proofs of Theorems 1.1 and 1.2 are given in Section 5. 


\section{The StRAightening MAP FOR HYPERBOLIC SIMPLICES}

In this section, we will prove a technical result (Lemma 2.3) that will be used in the subsequent sections. The proof of Lemma 2.3 makes use of both the hyperboloid and Klein models of $n$-dimensional hyperbolic space, which are now briefly reviewed.

2.1. The hyperboloid and Klein models of $\mathbb{H}^{n}$. Let $\mathbb{E}^{n, 1}$ be the Minkowski space which is $\mathbb{R}^{n+1}$ with inner product $\langle$,$\rangle defined by$

$$
\langle x, y\rangle=\sum_{i=1}^{n} x_{i} y_{i}-x_{n+1} y_{n+1},
$$

where $x=\left(x_{1}, \ldots, x_{n+1}\right)$ and $y=\left(y_{1}, \ldots, y_{n+1}\right)$. The hyperboloid model $H^{n}$ of $\mathbb{H}^{n}$ is

$$
H^{n}=\left\{x \in \mathbb{E}^{n, 1} \mid\langle x, x\rangle=-1, x_{n+1}>0\right\},
$$

and the light cone $\mathbb{L}$ is defined to be

$$
\mathbb{L}=\left\{x \in \mathbb{E}^{n, 1} \mid\langle x, x\rangle=0, x_{n+1}>0\right\} .
$$

Geodesics in the hyperboloid model $H^{n}$ are of the form $H^{n} \cap L$, where $L$ is a 2-dimensional linear subspace in $\mathbb{E}^{n, 1}$.

The open unit disc $D^{n} \subset \mathbb{R}^{n}$ is identified with a subset of $\mathbb{E}^{n, 1}$ via the natural maps

$$
D^{n} \subset \mathbb{R}^{n} \hookrightarrow \mathbb{R}^{n} \times\{1\} \subset \mathbb{E}^{n, 1} .
$$

The radial projection $\pi: H^{n} \rightarrow D^{n}$, defined by $\pi\left(x_{1}, \ldots, x_{n+1}\right)=\frac{1}{x_{n+1}}\left(x_{1}, \ldots, x_{n+1}\right)$, is a bijection, and the Klein model of the $n$-dimensional hyperbolic space is $D^{n}$ with the Riemannian metric induced from $H^{n}$ via $\pi$. Since $\pi$ maps geodesics in $H^{n}$ to line segments, geodesics in the Klein model $D^{n}$ are Euclidean straight lines. The boundary $\partial D^{n}$ of the Klein model $D^{n}$ is called the sphere at infinity, denoted by $S_{\infty}^{n-1}$, and $\overline{D^{n}}=D^{n} \cup S_{\infty}^{n-1}$ is called the compactification of $D^{n}$. We see that $\overline{D^{n}}$ naturally inherits a smooth structure from $\mathbb{R}^{n} \times\{1\} \subset \mathbb{E}^{n, 1}$.

We will denote

$$
\mathbb{E}_{<0}^{n, 1}=\left\{x \in \mathbb{E}^{n, 1} \mid\langle x, x\rangle<0, x_{n+1}>0\right\}
$$

and

$$
\mathbb{E}_{\leq 0}^{n, 1}=\left\{x \in \mathbb{E}^{n, 1} \mid\langle x, x\rangle \leq 0, x_{n+1}>0\right\} .
$$

We have $H^{n} \subset \mathbb{E}_{<0}^{n, 1} \subset \mathbb{E}_{\leq 0}^{n, 1}$ and the radial projection $\pi: H^{n} \rightarrow D^{n}$ can be viewed as the restriction to $H^{n}$ of the radial projection $\pi: \mathbb{E}_{\leq 0}^{n, 1} \rightarrow \overline{D^{n}}$.

\subsection{The straightening map for hyperbolic simplices. Let}

$$
\Delta^{k}=\left\{\left(t_{0}, \ldots, t_{k}\right) \in \mathbb{R}^{k+1} \mid t_{i} \geqslant 0, i=0, \ldots, k \text { and } \sum t_{i}=1\right\}
$$

be the standard $k$-simplex with vertex set $\left\{e_{i}\right\}$. Following [30], any $k+1$ points $v_{0}, \ldots, v_{k}, 1 \leqslant k \leqslant n$, in $D^{n}$ determine a straightening map (or straight $k$-simplex) $\sigma_{v_{0}, \ldots, v_{k}}: \Delta^{k} \rightarrow D^{n}$, whose image is the convex hull of $v_{0}, \ldots, v_{k}$. It is defined as follows. In the Minkowski space $\mathbb{E}^{n, 1}$, the $k+1$ points $u_{i}=\pi^{-1}\left(v_{i}\right) \in H^{n}$, $i \in\{0, \ldots, k\}$, determine an affine $k$-simplex $\sigma: \Delta^{k} \rightarrow \mathbb{E}_{<0}^{n, 1}$ by the barycentric coordinates

$$
\sigma\left(t_{0}, \ldots, t_{k}\right)=\sum_{i=0}^{k} t_{i} u_{i} .
$$


Definition 2.1. The straightening map $\sigma_{v_{0}, \ldots, v_{k}}: \Delta^{k} \rightarrow D^{n}$ is defined by $\sigma_{v_{0}, \ldots, v_{k}}$ $=\pi \circ \sigma$, and $\sigma: \Delta^{k} \rightarrow \mathbb{E}_{<0}^{n, 1}$ is called the affine simplex of $\sigma_{v_{0}, \ldots, v_{k}}$.

A straight ideal simplex is defined as follows. In this case, the vertices $v_{0}, \ldots, v_{k}$ are in $\partial D^{n}=S_{\infty}^{n-1}$. Choose $u_{0}, \ldots, u_{k}$ in the light cone $\mathbb{L}$ so that $\pi\left(u_{i}\right)=v_{i}$ for all $i$. Unlike the compact case, a preimage $u_{i}$ of the ideal vertex $v_{i}$ is not unique. Indeed, if $\pi\left(u_{i}\right)=v_{i}$, then $\pi^{-1}\left(v_{i}\right)=\left\{t \cdot u_{i} \mid t>0\right\}$. Let $\sigma_{u_{0}, \ldots, u_{k}}: \Delta^{k} \rightarrow \mathbb{E}_{\leq 0}^{n, 1}$ be the affine simplex defined by

$$
\sigma_{u_{0}, \ldots, u_{k}}\left(t_{0}, \ldots, t_{k}\right)=\sum_{i=0}^{k} t_{i} u_{i} .
$$

Then a straight ideal simplex with vertices $v_{0}, \ldots, v_{k}$ (with the choice of $u_{0}, \ldots, u_{k}$ ) is defined to be

$$
\pi \circ \sigma_{u_{0}, \ldots, u_{k}}: \Delta^{k} \rightarrow \overline{D^{n}} .
$$

In Penner's language [22, a straight ideal simplex is a hyperbolic ideal simplex with a horocycle assigned to each vertex, i.e., a decorated ideal simplex. Here is Penner's observation. Each vector $u \in \mathbb{L}$ defines a horocycle $\left\{x \in H^{n} \mid\langle x, u\rangle=-1\right\}$ at the ideal point $\pi(u)$ in $H^{n}$. In particular, given an ideal simplex with vertices $v_{0}, \ldots, v_{k}$, a choice of vectors $u_{0}, \ldots, u_{k}$ in the light cone $\mathbb{L}$ so that $\pi\left(u_{i}\right)=v_{i}$ for all $i$ means assigning a horocycle at each vertex $v_{i}$, i.e., a decorated ideal simplex. To summarize, the straightening map is defined for a decorated ideal simplex. A face of a decorated ideal simplex is considered as a decorated ideal simplex of lower dimension with the induced decoration of its vertices. In particular, a face of a straight ideal simplex is also straight in the above sense.

Proposition 2.2. The straight (and ideal straight) simplex is natural in the following sense. Suppose $\Delta$ is a compact or an ideal straight simplex with vertices $v_{0}, \ldots, v_{k}$ and $g \in \operatorname{Isom}\left(\mathbb{H}^{n}\right)$ is an isometry.

(1) If $\Delta^{\prime}$ is a face of a compact $k$-simplex $\Delta^{k}$ so that $\sigma_{v_{0}, \ldots, v_{k}}\left(\Delta^{\prime}\right)$ has vertices $v_{i_{0}}, \ldots, v_{i_{m}}$ in $H^{n}$, then

$$
\left.\sigma_{v_{0}, \ldots, v_{k}}\right|_{\Delta^{\prime}}=\sigma_{v_{i_{0}}, \ldots, v_{i_{m}}} .
$$

Furthermore,

$$
g \circ \sigma_{v_{0}, \ldots, v_{k}}=\sigma_{g \cdot v_{0}, \ldots, g \cdot v_{k}} .
$$

(2) If $\Delta^{\prime}$ is a face of an ideal straight simplex $\Delta^{k}$ so that $\sigma_{u_{0}, \ldots, u_{k}}\left(\Delta^{\prime}\right)$ has vertices $u_{i_{0}}, \ldots, u_{i_{m}}$ in $\mathbb{L}$, then

$$
\left.\pi \circ \sigma_{u_{0}, \ldots, u_{k}}\right|_{\Delta^{\prime}}=\pi \circ \sigma_{u_{i_{0}}, \ldots, u_{i_{m}}} .
$$

Furthermore,

$$
g \circ \pi \circ \sigma_{u_{0}, \ldots, u_{k}}=\pi \circ \sigma_{g \cdot u_{0}, \ldots, g \cdot u_{k}} .
$$

Proof. (1) is true since both sides of the equation equal $\left.\pi \circ \sigma\right|_{\Delta^{\prime}}$. To see the second part of (1), if $\sigma$ is the affine simplex of $\sigma_{v_{0}, \ldots, v_{k}}$, then $g \cdot \sigma$ is the affine simplex of $\sigma_{g \cdot v_{0}, \ldots, g \cdot v_{k}}$. Therefore, $\pi \circ g \cdot \sigma=\sigma_{g \cdot v_{0}, \ldots, g \cdot v_{k}}$. Since the radial projection commutes with each $g \in \operatorname{Isom}\left(\mathbb{H}^{n}\right), g \cdot \sigma_{v_{0}, \ldots, v_{k}}=g \cdot(\pi \circ \sigma)=\pi \circ g \cdot \sigma=\sigma_{g \cdot v_{0}, \ldots, g \cdot v_{k}}$. The proof of part (2) is similar. 
Lemma 2.3. Let $\left\{\sigma_{t}: \Delta^{k} \rightarrow D^{n} \mid t \in \mathbb{R}_{\geqslant 0}\right\}$ be a family of straight $k$-simplices so that the $i$-th vertex $v_{i, t}$ of $\sigma_{t}$ lies on the geodesic ray $l_{i}$ and $v_{i, t}$ moves toward the end-point $v_{i}^{*}$ of $l_{i}$ at unit speed, i.e., $d\left(v_{i, 0}, v_{i, t}\right)=t$. If $v_{0}^{*}, \ldots, v_{k}^{*}$ are pairwise distinct, then as $t$ tends to $\infty$ the family $\left\{\sigma_{t}\right\}$ converges pointwise to an ideal straight $k$-simplex $\sigma_{\infty}: \Delta^{k} \rightarrow \overline{D^{n}}$ whose vertices are $v_{0}^{*}, \ldots, v_{k}^{*}$. Furthermore, $\lim _{t \rightarrow \infty} \operatorname{Vol}\left(\sigma_{t}\right)=\operatorname{Vol}\left(\sigma_{\infty}\right)$.

Proof. The proof is based on the following observation. Suppose

$$
\gamma(t)=\left(\gamma_{1}(t), \ldots, \gamma_{n+1}(t)\right)
$$

for $t \in \mathbb{R}$ is a geodesic in the hyperboloid model $H^{n}$, which is parameterised by unit speed. Then for $j \in\{1, \ldots, n+1\}$,

$$
\lim _{t \rightarrow \infty} \frac{\gamma_{j}(t)}{\cosh (t)}
$$

exists and is in $[0,+\infty)$. Moreover, not all limits are zero. Indeed, the result is obvious for the specific geodesic $\delta(t)=(\sinh (t), 0, \ldots, 0, \cosh (t))$. Now any other geodesic $\gamma(t)$ is the image of $\delta(t)$ under a linear transformation (independent of $t$ ) preserving $H^{n}$.

To prove the proposition, for $\sigma_{t}$, let $\widetilde{\sigma_{t}}$ be the affine simplex in $\mathbb{E}^{n, 1}$ with the same set of vertices as that of $\sigma_{t}$. Then by definition $\sigma_{t}=\pi \circ \widetilde{\sigma}_{t}$. Let $\tau_{t}=\frac{1}{\cosh (t)} \cdot \widetilde{\sigma}_{t}$ be a new affine simplex. By the choice of vertices of $\widetilde{\sigma_{t}}$ and (2), the family of maps $\tau_{t}$ converges pointwise to an affine map $\tau_{\infty}: \Delta^{k} \rightarrow \mathbb{E}^{n, 1}$ so that the vertices of $\tau_{\infty}$ are mapped by $\pi$ to $v_{0}^{*}, \ldots, v_{k}^{*}$. It follows that $\sigma_{t}=\pi\left(\widetilde{\sigma_{t}}\right)=\pi\left(\tau_{t}\right)$ converges pointwise to an ideal straight $k$-simplex.

To see the convergence of the volume of $\sigma_{t}$ to $\sigma_{\infty}$, we observe that since the vertices converge, the corresponding dihedral angles of $\sigma_{t}$ converge to those of $\sigma_{\infty}$. Now by the solution of Milnor's conjecture on the volume of hyperbolic simplices [12, we conclude that the volumes converge.

\section{Thurston's SPINNING CONSTRUCTION}

Let $M$ be a closed hyperbolic 3-manifold, and $\mathcal{T}$ be a (possibly semi-simplicial) triangulation of $M$ with the property that all edges are essential. We denote by $M^{(k)}$ the $k$-skeleton in $M$. Let $p: \widetilde{M} \rightarrow M$ be the universal cover of $M$ and $\widetilde{\mathcal{T}}$ be the triangulation of $\widetilde{M}$ induced by $\mathcal{T}$. Since all edges in $M$ are essential, it follows that if $\sigma$ is a 3 -simplex in $M$, then any lift $\widetilde{\sigma}$ of $\sigma$ to $\widetilde{M}$ has four distinct vertices. We may identify $\widetilde{M}$ with the Klein model $D^{3}$ using the hyperbolic metric, and the natural action of $\pi_{1}(M)$ by deck transformations on $D^{3}$ is by isometries. Thurston's spinning construction in our context is summarized as follows.

Proposition 3.1. Let $M$ be a closed, triangulated, hyperbolic 3-manifold with the property that all edges in $M$ are essential. Then there exists a continuous family of piecewise smooth, $\pi_{1}(M)$-equivariant maps $\widetilde{F}_{t}: \widetilde{M} \rightarrow D^{3}, t \in[0, \infty)$, and a piecewise smooth, $\pi_{1}(M)$-equivariant map $\widetilde{F}_{\infty}: \widetilde{M} \rightarrow \overline{D^{3}}$, such that

(1) for each vertex $\widetilde{v}$ of $\widetilde{\mathcal{T}}$, the vertex $\widetilde{F}_{t}(\widetilde{v})$ approaches $S_{\infty}^{2}$, and for each $3-$ simplex $\widetilde{\sigma}$ of $\widetilde{\mathcal{T}}$ and each $t$ that is sufficiently large, $\widetilde{F}_{t}(\widetilde{\sigma})$ is a hyperbolic tetrahedron,

(2) $\widetilde{F}_{t}$ descends to a piecewise smooth map $F_{t}: M \rightarrow M$ which is homotopic to the identity map of $M$, for all $t \in[0, \infty)$, 
(3) $\widetilde{F}_{\infty}=\lim _{t \rightarrow \infty} \widetilde{F}_{t}$ pointwise in $\overline{D^{3}}$,

(4) for every 3 -simplex $\widetilde{\sigma}$ in $\widetilde{\mathcal{T}}, \widetilde{F}_{\infty}(\widetilde{\sigma})$ is an ideal tetrahedron with 4 distinct vertices in $\overline{D^{3}}$,

(5) $\widetilde{F}_{\infty}\left(\widetilde{M} \backslash p^{-1}\left(M^{(0)}\right)\right) \subset D^{3}$, and $\left.\widetilde{F}_{\infty}\right|_{\widetilde{M} \backslash p^{-1}\left(M^{(0)}\right)}$ descends to a piecewise smooth map $F_{\infty}: M \backslash M^{(0)} \rightarrow M$.

Proof. Suppose $M^{(0)}=\left\{v_{i}\right\}, i \in\left\{1, \ldots,\left|M^{(0)}\right|\right\}$. For each $v_{i}$, we will choose a geodesic ray $l_{i}$ starting at $v_{i}$ such that for every 3 -simplex $\widetilde{\sigma}$ in $\widetilde{M}$ any two lifts of geodesic rays in $L=\left\{l_{i}\right\}$ which start at different vertices of $\widetilde{\sigma}$ have no end-points in common. Indeed, pick a lift $\widetilde{v_{i}}$ for each $v_{i}$. Let

$$
F=\left\{x \in \partial D^{3} \mid \exists 1 \neq \gamma \in \pi_{1}(M): \gamma \cdot x=x\right\} .
$$

Since every non-trivial element in $\pi_{1}(M)$ has precisely two fixed points, $F$ is countable. Select $v_{1}^{*} \in \partial D^{3} \backslash F$. Then choose $v_{2}^{*} \in \partial D^{3} \backslash\left(F \cup \pi_{1}(M) \cdot\left\{v_{1}^{*}\right\}\right)$, and inductively choose $v_{i+1}^{*} \in \partial D^{3} \backslash\left(F \cup \pi_{1}(M) \cdot\left\{v_{1}^{*}, \ldots, v_{i}^{*}\right\}\right)$. Let $\widetilde{l_{i}}$ be the geodesic ray from $\widetilde{v_{i}}$ to $v_{i}^{*}$ and $l_{i}=p\left(\widetilde{l_{i}}\right)$. It is now easy to see that $L=\left\{l_{i}\right\}$ has the required property.

We parameterise each $l_{i}:[0, \infty) \rightarrow M$ by $l_{i}(0)=v_{i}$ and $\left\|l_{i}^{\prime}(t)\right\|_{\mathbb{H}^{3}}=1$ for all $t \in[0,+\infty)$. To construct $\widetilde{F}_{t}: \widetilde{M} \rightarrow D^{3}$, for each $i \in\left\{1, \ldots,\left|M^{(0)}\right|\right\}$, pick $\widetilde{v_{i}} \in p^{-1}\left(v_{i}\right)$ and a lift $\widetilde{l_{i}}$ of $l_{i}$ passing through $\widetilde{v_{i}}$, and define

$$
\widetilde{F}_{t}\left(\widetilde{v}_{i}\right)=\exp _{\widetilde{v_{i}}}\left(t \cdot \widetilde{l}_{i}^{\prime}(0)\right),
$$

where $\exp _{v}$ is the exponential map at $v$.

We define $\widetilde{F}_{t}: p^{-1}\left(M^{(0)}\right) \rightarrow D^{3}$ by

$$
\widetilde{F}_{t}(\gamma \cdot \widetilde{v})=\gamma \cdot \widetilde{F}_{t}(\widetilde{v}), \quad \widetilde{v} \in p^{-1}\left(M^{(0)}\right) \text { and } \gamma \in \pi_{1}(M),
$$

using the action of $\pi_{1}(M)$ on $\widetilde{M}$ and $D^{3}$ by deck transformations.

The map $\widetilde{F}_{t}$ is now extended to the 3 -simplices in $\widetilde{\mathcal{T}}$ by straightening maps. By part (1) of Propositon 2.2, this gives a well-defined map $\widetilde{F}_{t}: \widetilde{M} \rightarrow D^{3}$ since the straightening maps agree on intersections of 3 -simplices in $\widetilde{M}$. By part (1) of Proposition 2.2 the map $\widetilde{F}_{t}$ is $\pi_{1}(M)$-equivariant.

By equivariance, $\widetilde{F}_{t}$ descends to a piecewise smooth map

$$
F_{t}: M \rightarrow M \text {. }
$$

For any $t_{0} \in \mathbb{R}_{+}$, the map $H_{t_{0}}: M \times[0,1] \rightarrow M$ defined by

$$
H_{t_{0}}(x, t)=F_{t_{0} t}(x)
$$

provides a homotopy between $F_{0}$ and $F_{t_{0}}$.

Take the homotopy between $i d_{M}$ and $F_{0}$ to be the straight line homotopy. Namely, for $\widetilde{x} \in \widetilde{M} \cong D^{3}$, there is a unique geodesic segment $l_{\widetilde{x}}:[0,1] \rightarrow D^{3}$ such that $l_{\widetilde{x}}(0)=\widetilde{x}$ and $l_{\widetilde{x}}(1)=\widetilde{F}_{0}(\widetilde{x})$. The map $H_{0}: M \times[0,1] \rightarrow M$ defined by

$$
H_{0}(x, t)=p \circ l_{\widetilde{x}}(t),
$$

where $\widetilde{x} \in \widetilde{M}$ is any lift of $x$, provides a homotopy from $i d_{M}$ to $F_{0}$. This proves parts (1) and (2). 
We now define a $\pi_{1}(M)$-equivariant map

$$
\widetilde{F}_{\infty}: p^{-1}\left(M^{(0)}\right) \rightarrow \overline{D^{3}}
$$

as follows. For each $v \in M^{(0)}$, choose a lift $\widetilde{v} \in p^{-1}\left(M^{(0)}\right)$ and let $\widetilde{l}$ be the corresponding lift of the associated element of $L$. As $t$ tends to infinity, $F_{t}(\widetilde{v})=$ $\exp _{\widetilde{v}}\left(t \cdot \widetilde{l}^{\prime}(0)\right)$ approaches an end-point $\widetilde{v}^{*} \in S_{\infty}^{2}$ of $\widetilde{l}$. Define $\widetilde{F}_{\infty}(\widetilde{v})$ to be $\widetilde{v^{*}}$ and

$$
\widetilde{F}_{\infty}(\gamma \cdot \widetilde{v})=\gamma \cdot \widetilde{v}^{*}, \quad \gamma \in \pi_{1}(M) .
$$

By Lemma 2.3, on each simplex $\widetilde{\sigma}$ in $\widetilde{\mathcal{T}},\left\{\left.\widetilde{F}_{t}\right|_{\tilde{\sigma}}\right\}$ converges, so we can define

$$
\left.\left.\widetilde{F}_{\infty}\right|_{\tilde{\sigma}} \doteq \lim _{t \rightarrow \infty} \widetilde{F}_{t}\right|_{\tilde{\sigma}}
$$

and this agrees with the above definition on its vertices. By part (1) of Proposition 2.2, $\widetilde{F}_{t}$ is well-defined on intersections of 3 -simplices in $\widetilde{M}$, and hence is $\widetilde{F}_{\infty}$. Since each $\widetilde{F}_{t}$ is $\pi_{1}(M)$-equivariant, so is $\widetilde{F}_{\infty}$. This completes the proof of part (3).

By the assumption on the end-points of the set of lifted geodesics, the vertices of $\widetilde{F}_{\infty}(\widetilde{\sigma})$ are distinct. Therefore $\widetilde{F}_{\infty}(\widetilde{\sigma})$ is an ideal tetrahedron with four distinct vertices in $\overline{D^{3}}$. This proves (4), and (5) is a direct consequence of (4).

Remark 3.2. The choice of $L$ in the proof avoids the situation in [29], where simplices of higher dimension may be mapped into the sphere at infinity. In the language of [29], our subcomplex at infinity consists merely of the 0-skeleton.

\section{THE PARAMETER SPACE}

Throughout this section, we suppose that $M$ is a triangulated, oriented, closed 3 -manifold. We denote by $\Sigma^{k}$ the set of all $k$-simplices of the triangulation in $M$. As above, the triangulation may be semi-simplicial, so an element of $\Sigma^{k}$ may not be an embedded $k$-simplex in $M$. Nevertheless, elements of $\Sigma^{1}$ will be termed edges and elements of $\Sigma^{3}$ are termed tetrahedra.

4.1. The hyperbolic gluing equations. Let $\Delta^{3}$ be the standard 3-simplex with a chosen orientation. Suppose the edges from one vertex of $\Delta^{3}$ are labeled by $e_{1}$, $e_{2}$ and $e_{3}$ so that the opposite edges have the same labeling. Then the cyclic order of $e_{1}, e_{2}$ and $e_{3}$ viewed from each vertex depends only on the orientation of the 3 -simplex, i.e. is independent of the choice of the vertices. It follows that, up to orientation-preserving symmetries, there are two possible labelings, and we will fix one of these labelings.

Each pair of opposite edges corresponds to a normal isotopy class of quadrilaterals (normal quadrilateral for short) in $\Delta^{3}$. There is a natural cyclic order on the set of normal quadrilaterals induced by the cyclic order on the edges from a vertex, and this order is preserved by all orientation-preserving symmetries of $\Delta^{3}$.

If $\sigma \in \Sigma^{3}$, then there is an orientation-preserving map $\Delta^{3} \rightarrow \sigma$ taking the $k$-simplices in $\Delta^{3}$ to elements of $\Sigma^{k}$, and which is a bijection between the sets of normal quadrilaterals. This map induces a cyclic order of the normal quadrilaterals in $\sigma$, and we denote the corresponding $3-$ cycle $\tau_{\sigma}$. It follows from the above remarks, that this order is independent of the choice of the map. We define

$$
\tau=\prod_{\sigma \in \Sigma^{3}} \tau_{\sigma}
$$


Let $e \in \Sigma^{1}$, and $q$ be a normal quadrilateral in $\sigma$. The index $i(q, e)$ is the integer 0, 1 or 2 defined as follows: $i(q, e)=0$ if $e$ is not an edge of $\sigma, i(q, e)=1$ if $e$ is the only edge in $\sigma$ facing $q$, and $i(q, e)=2$ if $e$ are the two edges in $\sigma$ facing $q$.

Definition 4.1. The parameter space $\mathcal{P}(M)$ is the set of all points $Z=\left(z_{q}\right) \in$ $(\mathbb{C} \backslash\{0,1\})^{Q}$, where $Q$ is the set of all normal quadrilaterals, satisfying the following two conditions:

(a) for each edge $e$ in $M$,

$$
\prod_{q \in Q} z_{q}^{i(q, e)}=1
$$

(b) for each $q \in Q$,

$$
z_{\tau q}=\frac{1}{1-z_{q}}
$$

Equation (4) is the hyperbolic gluing equation of $e$, and (5) is the parameter relation of $q$.

Let $z_{\sigma}=\left(z_{q}, z_{\tau q}, z_{\tau^{2} q}\right)$ be the triple of complex numbers assigned to the three normal quadrilaterals in the tetrahedron $\sigma$. We will write $Z \in \mathcal{P}(M)$ as $Z=\left(z_{\sigma}\right)$.

4.2. The (lack of) geometry of solutions. In contrast to the case of cusped hyperbolic 3-manifolds, solutions to the hyperbolic gluing equations cannot be used to directly construct a hyperbolic metric on a closed 3-manifold:

Proposition 4.2. Let $M$ be a triangulated, closed, oriented $3-$ manifold, and $Z \in$ $\mathcal{P}(M)$. Then there is at least one 3 -simplex $\sigma \in \Sigma^{3}$ such that for any normal quadrilateral $q \in \sigma, z_{q} \in-\overline{\mathbb{H}}$, the closure of the lower half-plane.

Proof. Suppose for all $\sigma, z_{\sigma} \in \mathbb{H}$. Then by taking arguments, we would have obtained an angle structure on $M$, which is an assignment of real numbers, termed angles, in the range $(0, \pi)$ to each edge of each 3 -simplex such that the sum of all angles at each vertex of each 3-simplex is $\pi$, and such that around each edge $e$ of $\mathcal{T}$ the sum of angles is $2 k_{e} \pi$ with $k_{e} \geq 1$. This induces a combinatorial angle structure on the link of each vertex in $M$, that is, a function $a:\{$ all corners in the link $\} \rightarrow \mathbb{R}$. Since all 2-cells in the induced triangulation of the vertex link are triangles and have angle sum $\pi$, the total combinatorial area $A(a)$ of the angle structure is zero. The combinatorial curvature $K_{v}$ at vertex $v$ of the induced triangulation is $2 \pi$ minus the sum of angles at $v$, which equals $2\left(1-k_{e}\right) \pi$, where $e$ is the edge containing $v$, whence $K_{v}$ is non-positive. Since the link of each vertex in $M$ is a sphere, the combinatorial Gauß-Bonnet formula (see [14, Proposition 13) now implies that

$$
4 \pi=2 \pi \chi\left(S^{2}\right)=A(a)+\sum K_{v} \leq 0,
$$

which gives a contradiction.

The above result is sharp in the sense that there may not be any negatively oriented tetrahedra. For instance, the one-tetrahedron triangulation of $L(4,1)$ yields the unique solution $\left(-1, \frac{1}{2}, 2\right)$. Taking the arguments of $Z \in \mathcal{P}(M)$ defines an $S^{1}-$ angle structure on the closed 3-manifold $M$, which is the counterpart of an angle structure on a 3 -manifold with torus cusps (see [13] for results on $S^{1}$-angle structures). 
4.3. The volume of solutions. The Lobachevsky function is defined by:

$$
\Lambda(\alpha)=-\int_{0}^{\alpha} \ln |2 \sin t| d t
$$

for any $\alpha \in \mathbb{R}$.

Definition 4.3. The volume of $z_{\sigma}$ is defined to be the sum of the Lobachevsky functions

$$
\operatorname{Vol}\left(z_{\sigma}\right)=\Lambda\left(\arg \left(z_{q}\right)\right)+\Lambda\left(\arg \left(z_{\tau q}\right)\right)+\Lambda\left(\arg \left(z_{\tau^{2} q}\right)\right)
$$

and the volume of $\left(z_{\sigma}\right)=Z \in \mathcal{P}(M)$ is defined by

$$
\operatorname{Vol}(Z)=\sum_{\sigma \in \Sigma^{3}} \operatorname{Vol}\left(z_{\sigma}\right)
$$

The hyperbolic gluing equations are defined over the integers, so $Z \in \mathcal{P}(M)$ implies $\bar{Z} \in \mathcal{P}(M)$, where the latter point is obtained by taking the complex conjugates of all coordinates. It follows from the properties of the Lobachevsky function (see [19]) that $\operatorname{Vol}(Z)=-\operatorname{Vol}(\bar{Z})$.

4.4. The shape parameters of an ideal tetrahedron. Let $\sigma$ be an ideal geodesic tetrahedron in $\mathbb{H}^{3}$ with vertices $\left\{v_{i}\right\} \subset S_{\infty}^{2}, i=1, \ldots, 4$. The order $\left(v_{1}, \ldots, v_{4}\right)$ determines an orientation of $\sigma$. We call $\sigma$ positive if the orientation of $\sigma$ coincides with the orientation of $\mathbb{H}^{3}$, negative if the orientation of $\sigma$ differs from the one of $\mathbb{H}^{3}$, and flat if $\sigma$ lies in a totally geodesic plane.

Definition 4.4. Let $e_{i j}$ be the edge from $v_{i}$ to $v_{j}$, and identify $S_{\infty}^{2}$ with $\mathbb{C} \cup\{\infty\}$. Then the shape parameter of $\sigma$ at $e_{i j}$ (or edge invariant at $e_{i j}$ ) is defined by the cross-ratio

$$
\begin{aligned}
z_{i j} & \doteq\left(v_{i}, v_{j} ; v_{k}, v_{l}\right) \\
& =\frac{v_{i}-v_{k}}{v_{i}-v_{l}} \cdot \frac{v_{j}-v_{l}}{v_{j}-v_{k}},
\end{aligned}
$$

where $(i, j, k, l)$ is an even permutation of $(1,2,3,4)$.

A direct cross-ratio calculation shows the following well-known:

Proposition 4.5. With the above notation:

(1) For all $i \neq j: z_{i j}=z_{j i}$.

(2) For all $\{i, j\} \cup\{k, l\}=\{1,2,3,4\}$,

$$
z_{i j}=z_{k l},
$$

so opposite edges share the same shape parameter, and we can denote the shape parameter of $\sigma$ at $e_{i j}$ and $e_{k l}$ by $z_{q}$, where $q$ is the normal quadrilateral facing $e_{i j}$ and $e_{k l} ;$ and

(3) if the 3 -cycle $\tau_{\sigma}$ determines the cyclic order of the normal quadrilaterals in $\sigma$, then

$$
z_{\tau q}=\frac{1}{1-z_{q}}
$$

For an ideal tetrahedron $\sigma$ with shape parameters $z_{q}, z_{\tau q}$ and $z_{\tau^{2} q}$, the hyperbolic volume is calculated by Milnor as

$$
\operatorname{Vol}(\sigma)=\Lambda\left(\arg \left(z_{q}\right)\right)+\Lambda\left(\arg \left(z_{\tau q}\right)\right)+\Lambda\left(\arg \left(z_{\tau^{2} q}\right)\right) .
$$


Therefore, when $\sigma$ is positive, its shape parameters are in the upper half-plane and $\operatorname{Vol}(\sigma)>0$; when $\sigma$ is flat, its shape parameters are real and $\operatorname{Vol}(\sigma)=0$; when $\sigma$ is negative, its shape parameters are in the lower half-plane and $\operatorname{Vol}(\sigma)<0$.

4.5. The associated representation. The following is essentially the construction described by Yoshida for cusped 3-manifolds in [31], §5, though we will take more care of details which are needed for our application.

We assume that the triangulation of $M$ consists of a pairwise disjoint union of standard 3-simplices, $\widetilde{\Delta}=\bigcup_{k=1}^{n} \widetilde{\Delta}_{k}$, together with a collection $\Phi$ of Euclidean isometries between standard 2 -simplices in $\widetilde{\Delta}$; termed face pairings. Then $M=$ $\widetilde{\Delta} / \Phi$. Since $M$ is oriented, we may assume that all 3 -simplices in $\widetilde{\Delta}$ are coherently oriented, so that each face pairing is orientation-reversing.

Denote by $p: \widetilde{M} \rightarrow M$ the universal cover of $M$. Lift the triangulation of $M$ to a $\pi_{1}(M)$-equivariant triangulation of $\widetilde{M}$. Since each edge is essential, every tetrahedron in $\widetilde{M}$ is embedded. Let $Z \in \mathcal{P}(M)$. Then a map

$$
D_{Z}: \widetilde{M}^{(0)} \rightarrow \partial D^{3}
$$

can be defined inductively as follows.

Pick an oriented 3-simplex, say $\sigma=\left[e_{0}, e_{1}, e_{2}, e_{3}\right]$ in $\widetilde{M}$. It inherits a well-defined shape parameter $z_{\sigma}$ from $\sigma$. Choose $v_{0}, v_{1}, v_{2}, v_{3} \in \partial D^{3}$ such that the cross-ratio $z_{i j}=\left(v_{i}, v_{j} ; v_{k}, v_{l}\right)$ agrees with the shape parameter of $\sigma$ at edge $\left[e_{i}, e_{j}\right]$. Then define $D_{Z}\left(e_{i}\right)=v_{i}$ for $i \in\{0,1,2,3\}$.

Now suppose $D_{Z}$ is defined on the vertices of a triangulated subset $W$ of $\widetilde{M}$. Let $\sigma^{3}$ be a 3 -simplex in $\widetilde{M}$ which shares at least a 2 -simplex, say $\sigma^{2}$, with $W$. Suppose $\sigma^{3}=\left[w_{0}, w_{1}, w_{2}, w_{3}\right]$ and $\sigma^{2}=\left[w_{0}, w_{1}, w_{2}\right]$. Then define $D_{Z}\left(w_{3}\right) \in \partial D^{3}$ such that the cross-ratio

$$
\left(D_{Z}\left(w_{0}\right), D_{Z}\left(w_{1}\right) ; D_{Z}\left(w_{2}\right), D_{Z}\left(w_{3}\right)\right)
$$

equals the edge invariant of $\sigma^{3}$ at $\left[w_{0}, w_{1}\right]$. Even if $w_{3} \in W$, this is well-defined since the hyperbolic gluing equations are satisfied.

There is a natural isomorphism $\pi_{1}\left(M \backslash M^{(0)}\right) \cong \pi_{1}(M)$ by the Seifert-Van Kampen theorem. For each $\gamma \in \pi_{1}(M)$, there is a unique element $\rho_{Z}(\gamma) \in P S L_{2}(\mathbb{C})$ such that

$$
D_{Z}(\gamma x)=\rho_{Z}(\gamma) D_{Z}(x)
$$

for all $x \in \widetilde{M}^{(0)}$. To see this, define $\rho_{Z}(\gamma)$ to be the isometry which maps $D_{Z}\left(\sigma^{(0)}\right)$ to $D_{Z}\left(\gamma \sigma^{(0)}\right)$ for any 3 -simplex $\sigma$ in $\widetilde{M}$. This is well-defined since the hyperbolic gluing equations are satisfied. We thus have a representation $\rho_{Z}: \pi_{1}(M) \rightarrow P S L_{2}(\mathbb{C})$, which is uniquely determined by the map $D_{Z}$. The only choice in the construction of $D_{Z}$ is the initial placement of a 3 -simplex; a different choice results in a representation, which is conjugate to $\rho_{Z}$ by an orientation-preserving isometry of $\mathbb{H}^{3}$.

4.6. Representation volume. Given the closed 3-manifold $M$ and any representation $\rho: \pi_{1}(M) \rightarrow P S L_{2}(\mathbb{C})$, the volume of $\rho$ is defined as follows (see Dunfield [7] for details). Choose any piecewise smooth $\rho$-equivariant map $f: \widetilde{M} \rightarrow \mathbb{H}^{3}$. The form $f^{*}\left(d \mathrm{Vol}_{\mathbb{H}^{3}}\right)$ descends to a form on $M$. The volume of $\rho$ is the value of the integral of this form over $M$ :

$$
\operatorname{Vol}(\rho)=\int_{M} f^{*}\left(d \operatorname{Vol}_{\mathbb{H}^{3}}\right)
$$


The volume is independent of $f$ as any two such maps are equivariantly homotopic by a straight line homotopy (cf. the proof of Proposition 3.1). The above is a slight modification of Dunfield's definition in that he takes the absolute value of the integral, whilst we maintain dependence on the orientation of $M$.

With the notation of the previous subsection, we have the following result:

Lemma 4.6. Let $M$ be a closed, oriented, triangulated 3-manifold with the property that all edges in $M$ are essential. Then $\operatorname{Vol}\left(\rho_{Z}\right)=\operatorname{Vol}(Z)$ for each $Z \in \mathcal{P}(M)$.

The proof of the lemma is given in Section [5] below. Dunfield [7] proves the following rigidity result for representation volume, which he attributes to Thurston, Gromov and Goldman:

Theorem 4.7 (Thurston-Gromov-Goldman). If $M$ is a closed, oriented hyperbolic 3-manifold, and $\rho: \pi_{1}(M) \rightarrow P S L_{2}(\mathbb{C})$ a representation with $\operatorname{Vol}(\rho)=\operatorname{Vol}(M)$, then $\rho$ is discrete and faithful, and the quotient $\mathbb{H}^{3} / \rho\left(\pi_{1}(M)\right)$ is isometric to $M$.

Dunfield [7] in fact proves slightly more (see [9] for a further generalisation):

Proposition 4.8. Let $M$ be a closed, oriented hyperbolic 3-manifold, and suppose $\rho: \pi_{1}(M) \rightarrow P S L_{2}(\mathbb{C})$ is a representation of the fundamental group of $M$. Then $|\operatorname{Vol}(\rho)| \leq \operatorname{Vol}(M)$. Moreover, if $\operatorname{Vol}(\rho)= \pm \operatorname{Vol}(M)$, then $\rho$ is discrete and faithful, and the quotient $\mathbb{H}^{3} / \rho\left(\pi_{1}(M)\right)$ is isometric to $M$.

Putting Lemma 4.6 and Proposition 4.8 together, we have:

Corollary 4.9. Let $M$ be a closed, oriented, triangulated, hyperbolic 3-manifold with the property that all edges in $M$ are essential. Then $|\operatorname{Vol}(Z)| \leq \operatorname{Vol}(M)$ for each $Z \in \mathcal{P}(M)$, and if $\operatorname{Vol}(Z)= \pm \operatorname{Vol}(M)$, then $\rho_{Z}$ is discrete and faithful, and the quotient $\mathbb{H}^{3} / \rho_{Z}\left(\pi_{1}(M)\right)$ is isometric to $M$.

If $M$ is not hyperbolic, the range of Vol depends on both the pieces of the JSJ decomposition of $M$ and the way they glue up. The proof of Theorem 1.3 in [8] implies the following:

Lemma 4.10 (Francaviglia). Suppose the closed, orientable 3-manifold $M$ is a Seifert fibred space or a graph manifold. Then for any representation $\rho: \pi_{1}(M) \rightarrow$ $P S L_{2}(\mathbb{C})$, we have $\operatorname{Vol}(\rho)=0$.

\section{Proofs}

5.1. Proof of Lemma 4.6. Let $M$ be a closed, oriented, triangulated 3-manifold with the property that all edges in $M$ are essential; that is, we have dropped the hypothesis that $M$ be hyperbolic. We need to show that $\operatorname{Vol}\left(\rho_{Z}\right)=\operatorname{Vol}(Z)$ for each $Z \in \mathcal{P}(M)$.

Let $p: \widetilde{M} \rightarrow M$ be the universal cover of $M$, and use the setup from Subsection 4.5. To begin with, construct any $\rho_{Z}$-equivariant map $F_{0}: \widetilde{M} \rightarrow D^{3}$ with the property that every standard 3 -simplex in $\widetilde{M}$ is mapped by a straight map to $D^{3}$. Any such map can be constructed by first choosing a representative for each $\pi_{1}(M)$-orbit of vertices in $\widetilde{M}$, as well as an image point in $D^{3}$ for each orbit representative. One then extends the map over the whole 0 -skeleton $\rho_{Z}$-equivariantly, and over the 3-skeleton by straight maps. This is clearly well-defined given our rigid setup. 
Let $\sigma^{0}$ be a vertex in $\widetilde{M}$, and suppose $D_{Z}\left(\sigma^{0}\right)=v$. Then let $l_{\sigma^{0}}$ be the geodesic ray from $F_{0}\left(\sigma^{0}\right)$ to $v$. This gives a set of geodesic rays, one for each vertex in $\widetilde{M}$. Since both maps, $F_{0}$ and $D_{Z}$, are $\rho_{Z}$-equivariant, the set of rays is also $\rho_{Z^{-}}$ equivariant.

For each $t \in(0, \infty)$, define the map $F_{t}: \widetilde{M} \rightarrow D^{3}$ as follows. If $\sigma^{0}$ is a vertex in $\widetilde{M}$, then let $F_{t}\left(\sigma^{0}\right)$ be the point on the ray $l_{\sigma^{0}}$ which is at a distance $t$ from $F_{0}\left(\sigma^{0}\right)$. Then extend $F_{t}$ to the 3 -simplices by straight maps. Since the action of $\pi_{1}(M)$ on $D^{3}$ via $\rho_{Z}$ is by isometries, this is a well-defined, $\rho_{Z}$-equivariant map. Since $D_{Z}$ maps the vertices of every 3 -simplex to four distinct points on $\partial D^{3}$, i.e., the vertices of an ideal hyperbolic 3-simplex (possibly flat), we are in the situation of Lemma 2.3. It follows that there is a $\rho_{Z}$-equivariant map $F_{\infty}: \widetilde{M} \rightarrow \overline{D^{3}}$ such that

$$
F_{\infty}=\lim _{t \rightarrow \infty} F_{t} \quad \text { and }\left.\quad F_{\infty}\right|_{\widetilde{M}^{(0)}}=D_{Z}
$$

For each $\sigma \in \Sigma^{3}$, choose a standard 3-simplex $\widetilde{\sigma} \subset p^{-1}(\sigma)$. We have

$$
\begin{aligned}
\operatorname{Vol}(Z) & =\sum_{\sigma \in \Sigma^{3}} \operatorname{Vol}\left(z_{\sigma}\right) \\
& =\sum_{\sigma \in \Sigma^{3}} \operatorname{Vol}_{\mathbb{H}^{3}}\left(F_{\infty}(\widetilde{\sigma})\right) \\
& =\int_{M \backslash M^{(0)}}\left(F_{\infty}\right)^{*}\left(d \operatorname{Vol}_{\mathbb{H}^{3}}\right) \\
& =\int_{M}\left(\lim _{t \rightarrow \infty} F_{t}\right)^{*}\left(d \operatorname{Vol}_{\mathbb{H}^{3}}\right) \\
& =\lim _{t \rightarrow \infty} \int_{M}\left(F_{t}\right)^{*}\left(d \operatorname{Vol}_{\mathbb{H}^{3}}\right) \\
& =\operatorname{Vol}\left(\rho_{Z}\right)
\end{aligned}
$$

since each term in the limit is constant.

5.2. Proof of Theorem 1.1. Let $M$ be a closed, oriented, triangulated, hyperbolic 3 -manifold with the property that all edges in $M$ are essential. It follows from Corollary 4.9 that whenever $\operatorname{Vol}(Z)= \pm \operatorname{Vol}(M)$, then $\rho_{Z}$ is discrete and faithful. In this case, Mostow rigidity implies that $M$ is isometric with $\mathbb{H}^{3} / \rho_{Z}\left(\pi_{1}(M)\right)$, where the isometry is orientation-preserving if $\operatorname{Vol}(Z)=\operatorname{Vol}(M)$, and orientationreversing if $\operatorname{Vol}(Z)=-\operatorname{Vol}(M)$. It remains to prove the existence of a maximum volume solution. This follows from an analysis of the map $\widetilde{F}_{\infty}$ of Proposition 3.1

Let $\sigma$ be a 3 -simplex in $M$, and $\widetilde{\sigma}$ a lift of $\sigma$ in $\widetilde{M}$. By (4) of Proposition 3.1, $\widetilde{\sigma}^{*} \doteq \widetilde{F}_{\infty}(\widetilde{\sigma})$ is a non-degenerate ideal tetrahedron in $\overline{D^{3}}$ and has the associated shape parameters $Z_{\widetilde{\sigma}^{*}}=\left(z_{q_{i}}\right), i=1,2,3$. For any 3 -simplex $\sigma$ in $M$, we assign the shape parameters $z_{q_{i}}$ of $\widetilde{\sigma}^{*}$ to the corresponding $q_{i} \subset \sigma$ and get an assignment $Z_{\infty}=\left(z_{\sigma}\right) \in(\mathbb{C} \backslash\{0,1\})^{Q}$. We claim that $Z_{\infty}$ is a solution to the hyperbolic gluing equation, i.e., $Z_{\infty}$ satisfies (a) and (b) of Definition 4.1. Indeed, by (2) of Proposition 4.5 (b) is obvious.

To show (a), let $e$ be an edge in $\mathcal{T}$, and $\widetilde{e}$ a lift of $e$ in $\widetilde{\mathcal{T}}$ with end-points $\widetilde{u}$ and $\widetilde{w}$. Let $\widetilde{\sigma}_{1}, \ldots, \widetilde{\sigma}_{k}$ be the tetrahedra in $\widetilde{\mathcal{T}}$ having $\widetilde{e}$ as an edge in cyclic order and $\widetilde{q}_{i}$ be the normal quadrilateral in $\widetilde{\sigma}_{i}$ facing $\widetilde{e}$. Let $\widetilde{v}_{i}$ and $\widetilde{v}_{i+1}$ be the other 
two vertices of $\widetilde{\sigma}_{i}$ so that $\widetilde{v}_{i} \in \widetilde{\sigma}_{i-1} \cap \widetilde{\sigma}_{i}$. By (4) of Proposition 3.1 we get $k$ ideal tetrahedra $\widetilde{\sigma}_{i}^{*}=\widetilde{F}_{\infty}\left(\widetilde{\sigma}_{i}\right)$ sharing the geodesic $\widetilde{e}^{*}=\widetilde{F}_{\infty}(\widetilde{e})$ as an edge. Without loss of generality, we may assume that $\widetilde{e}^{*}$ is the geodesic from 0 to $\infty$. Suppose $z_{i}$ is the complex number assigned to the normal quadrilateral $q_{i}$ in $\sigma_{i}$ facing $e$, i.e., the shape parameter of $\widetilde{\sigma}_{i}^{*}$ at the normal quadrilateral $\widetilde{q}_{i}$. We have

$$
\prod_{q \in Q} z_{q}^{i(q, e)}=\prod_{i=1}^{k} z_{\widetilde{q}_{i}}=\prod_{i=1}^{k}\left(0, \infty ; \widetilde{v}_{i}^{*}, \widetilde{v}_{i+1}^{*}\right)=\prod_{i=1}^{k} \frac{\widetilde{v}_{i}^{*}}{\widetilde{v}_{i+1}^{*}}=1,
$$

where the first equality follows from the definition of $Z_{\infty}$, the second from Definition 4.4 and $\widetilde{v}_{k+1}^{*}$ is understood to be $\widetilde{v}_{1}^{*}$. This verifies (a).

To prove the volume identity, we have the following calculation:

$$
\begin{array}{rlrl}
\operatorname{Vol}\left(Z_{\infty}\right) & =\sum_{\sigma \in \Sigma^{3}} \operatorname{Vol}\left(z_{\sigma}\right) & \\
& =\sum_{\sigma \in \Sigma^{3}} \operatorname{Vol}_{\mathbb{H}^{3}}\left(\widetilde{\sigma}_{\infty}\right) & & \text { by Definition } 4.3 \text { and (66) } \\
& =\int_{M \backslash M^{(0)}}\left(F_{\infty}\right)^{*}\left(d \operatorname{Vol}_{\mathbb{H}^{3}}\right) & & \text { by (4) of Proposition 3.1 } \\
& =\int_{M}\left(\lim _{t \rightarrow \infty} F_{t}\right)^{*}\left(d \operatorname{Vol}_{\mathbb{H}^{3}}\right) & & \text { by (3) of Proposition 3.1 } \\
& =\lim _{t \rightarrow \infty} \int_{M}\left(F_{t}\right)^{*}\left(d \operatorname{Vol}_{\mathbb{H}^{3}}\right) & & \text { by Lemma 2.3. }
\end{array}
$$

By (5) of Proposition 3.1, $F_{t}$ is homotopic to the identity map of $M$, so we have

$$
\lim _{t \rightarrow \infty} \int_{M} F_{t}^{*}\left(d \operatorname{Vol}_{\mathbb{H}^{3}}\right)=\lim _{t \rightarrow \infty} \int_{M} d \operatorname{Vol}_{M}=\operatorname{Vol}(M) .
$$

This completes the proof.

5.3. Proof of Theorem 1.2. One first needs to decide whether $M$ is irreducible and atoroidal. The fact that this can be done follows from the work of Haken; see Jaco and Tollefson [1] for a complete exposition.

So we may suppose that $M$ is a triangulated, closed, irreducible, atoroidal and oriented 3-manifold. It follows from Thurston's Geometrisation Conjecture (which now is a complete theorem due to Perelman et al. [23, 25, 24, 20, 21, 15, 44) that $M$ is either hyperbolic or a small Seifert fibred space. By passing to a barycentric subdivision, we may assume that all edges are essential.

The parameter space has finitely many Zariski components and the volume function is constant on each component. (In fact, it is constant on topological components.) Hence pick one point from each component. If Vol vanishes on each or if the parameter space is empty, then it follows from Theorem 1.1 that $M$ is not hyperbolic, whence it is a small Seifert fibred space. In this case, Sela [28], Section 10, describes an effective algorithm to recognise the Seifert fibred structure using the fundamental group, and the classification of Seifert fibred spaces can be used to construct the structure.

Hence suppose that there is a point $Z \in \mathcal{P}(M)$ with $\operatorname{Vol}(Z) \neq 0$. It follows from Lemma 4.10 that $M$ cannot be a small Seifert fibred space and hence is hyperbolic. 
Theorem 1.1 implies that the values of $\operatorname{Vol}$ lie in $[-\operatorname{Vol}(M), \operatorname{Vol}(M)]$ and that both bounds are attained. Then a discrete and faithful representation of $\pi_{1}(M)$ into $P S L_{2}(\mathbb{C})$ is the holonomy representation determined by a point of maximal volume. The structure can now be constructed as described by Manning (see [17, Section 4).

It remains to address how to turn the above outline into a rigorous algorithm. The first part, checking that $M$ is irreducible and atoroidal using normal surface theory, is clearly rigorous. The routines required to carry out the necessary steps have been described by Jaco and Tollefson [11, and they are all implemented in Regina [3. In fact, there is a specific routine in Regina, which will check whether a manifold is irreducible. Once this has been answered in the affirmative, one can use Regina to compute the vertex normal tori. The manifold is toroidal if and only if one of these is incompressible. To check incompressibility of such a torus, one cuts the manifold along the torus. This gives a triangulated manifold with boundary. If this manifold is connected, then the torus must be incompressible. Otherwise, one enumerates the vertex normal surfaces of each component to decide whether or not there is a compression disc for the surface.

The algorithms from algebraic geometry which are needed involve computations with algebraic numbers over the rationals. Manning (see [17, Section 2) has summarised most of what we need using results from [1] and 16, including prime decomposition and determining the dimension of an ideal. The ability to pick a point from each Zariski component of positive dimension using exact arithmetic follows from the fact that in order to determine dimension, one finds a maximally independent set of coordinates. Each is a non-constant function on the variety, and one can therefore use standard elimination theory (see for instance [6]) to determine a point on the Zariski component algorithmically.

Having one point from each Zariski component, say $\left\{Z_{1}, \ldots, Z_{k}\right\}$, it suffices to compute $\operatorname{Vol}\left(Z_{i}\right)$ up to high enough precision in order to determine the maximum point as follows. First compute $\operatorname{Vol}\left(Z_{i}\right)$ up to a pre-determined precision. If each $\operatorname{Vol}\left(Z_{i}\right)$ is estimated to be less than 0.9 , then the maximum must be equal to zero, since recent work of Gabai, Meyerhoff and Milley [10, 18] has shown that the Weeks manifold (which has volume approximately 0.9427 ) is the orientable hyperbolic 3-manifold of smallest volume. Otherwise, compare the values to determine the maximum. In the event that two or more values are equal at the pre-determined precision, one can compute the characters of the associated representations in exact arithmetic. If they agree, either of the points will be the desired maximum. If they do not, the procedure of incrementally increasing the precision will eventually terminate. This algorithm is to a point not only theoretical, but also practical: computing volume up to any given precision (but subject to hardware limitations) is implemented in the software snap [5].

\section{REFERENCES}

1. Thomas Becker and Volker Weispfenning: Gröbner Bases: A Computational Approach to Commutative Algebra, Springer-Verlag, New York, 1993. MR.1213453 (95e:13018)

2. Riccardo Benedetti and Carlo Petronio: Lectures on Hyperbolic Geometry. Springer-Verlag, Berlin, 1992. MR.1219310 (94e:57015)

3. Benjamin A. Burton: Regina: Normal Surface and 3-Manifold Topology Software, 1999-2009, http://regina.sourceforge.net/. 
4. Huai-Dong Cao and Xi-Ping Zhu: A complete proof of the Poincaré and geometrization conjectures-application of the Hamilton-Perelman theory of the Ricci flow, Asian J. Math. 10 (2006), no. 2, 165-492; Erratum, Asian J. Math. 10 (2006), no. 4, p. 663. MR2233789 (2008d:53090), MR2282358 (2008d:53091)

5. David Coulson, Oliver A. Goodman, Craig D. Hodgson and Walter D. Neumann: Computing arithmetic invariants of 3-manifolds, Experimental Mathematics 9 (2000), 127-152. MR:1758805 (2001c:57014)

6. David Cox, John Little and Donal O'Shea: Ideals, varieties, and algorithms. An introduction to computational algebraic geometry and commutative algebra. Third edition. Springer, New York, 2007. MR,2290010 (2007h:13036)

7. Nathan M. Dunfield: Cyclic surgery, degrees of maps of character curves, and volume rigidity for hyperbolic manifolds, Invent. Math. 136 (1999), no. 3, 623-657. MR1695208(2000d:57022)

8. Stefano Francaviglia: Hyperbolic volume of representations of fundamental groups of cusped 3-manifolds, IMRN, 2004, no. 9, 425-459. MR2040346 (2004m:57032)

9. Stefano Francaviglia and Ben Klaff: Maximal volume representations are Fuchsian, Geom. Dedicata 117 (2006), 111-124. MR2231161 (2007d:51019)

10. David Gabai, Robert Meyerhoff and Peter Milley: Minimum volume cusped hyperbolic three-manifolds, Journal of the American Mathematical Society 22 (4) (2009), 1157-1215. MR2525782 (2011a:57031)

11. William Jaco and Jeffrey Tollefson: Algorithms for the complete decomposition of a closed 3-manifold, Illinois J. Math. 39 (1995), no. 3, 358-406. MR.1339832 (97a:57014)

12. Feng Luo: Continuity of the volume of simplices in classical geometry, Commun. Contemp. Math. 8 (2006), no. 3, 411-431. MR2230889 (2007f:52026)

13. Feng Luo: Volume optimization, normal surfaces and Thurston's equation on triangulated 3-manifolds, Preprint, arXiv:0903.1138v1.

14. Feng Luo and Stephan Tillmann: Angle structures and normal surfaces, Trans. Amer. Math. Soc. 360 (2008), no. 6, 2849-2866. MR2379778 (2009b:57046)

15. Bruce Kleiner and John Lott: Notes on Perelman's papers, Geom. Topol. 12 (2008), 25872855. MR2460872 (2010h:53098)

16. Rüdiger Loos: Computing in Algebraic Extensions, from: "Computer Algebra: Symbolic and Algebraic Computation" (Bruno Buchberger, George Edwin Collins, Rüdiger Loos, Rudolf Albrecht, editors), Springer-Verlag, Vienna, 1983, 173-188. MR728972

17. Jason Manning: Algorithmic detection and description of hyperbolic structures on closed 3-manifolds with solvable word problem, Geometry \& Topology 6 (2002) 1-26. MR 1885587 (2002k:57043)

18. Peter Milley: Minimum volume hyperbolic 3-manifolds, Journal of Topology 2 (2009), no. 2, 181-192. MR2499442(2010d:57018)

19. John Milnor: Collected papers. Vol. 1. Geometry. Publish or Perish, Inc., Houston, TX, 1994. MR1277810 (95c:01043)

20. John Morgan and Gang Tian: Ricci flow and the Poincaré conjecture, AMS, Providence, 2007. MR2334563 (2008d:57020)

21. John Morgan and Gang Tian: Completion of the Proof of the Geometrization Conjecture, arXiv:0809.4040v1.

22. Robert Penner: The decorated Teichmüller space of punctured surfaces, Comm. Math. Phys. 113 (1987), no. 2, 299-339. MR919235(89h:32044)

23. Grisha Perelman: The entropy formula for the Ricci flow and its geometric applications, arXiv:math/0211159.

24. Grisha Perelman: Finite extinction time for the solutions to the Ricci flow on certain threemanifolds, arXiv:math/0307245.

25. Grisha Perelman: Ricci flow with surgery on three-manifolds, arXiv:math/0303109.

26. John G. Ratcliffe: Foundations of hyperbolic manifolds. Second edition, Graduate Texts in Mathematics, 149. Springer, New York, 2006. MR2249478 (2007d:57029)

27. J. Hyam Rubinstein: An algorithm to recognise small Seifert fiber spaces, Turkish J. Math. 28 (2004), 75-87. MR2056761 (2005b:57042)

28. Zlil Sela: The isomorphism problem for hyperbolic groups I, Annals of Mathematics (2) 141 (1995), 217-283. MR1324134 (96b:20049)

29. William P. Thurston: Hyperbolic structures on 3-manifolds I: Deformation of acylindrical manifolds, Annals of Mathematics (2) 124 (1986), no. 2, 203-246. MR855294 (88g:57014) 
30. William P. Thurston: The geometry and topology of 3-manifolds, Princeton Univ. Math. Dept. (1978). Available from http://msri.org/publications/books/gt3m/.

31. Tomoyoshi Yoshida: On ideal points of deformation curves of hyperbolic 3-manifolds with one cusp, Topology 30 (1991), no. 2, 155-170. MR.1098911(92a:57018)

Department of Mathematics, Rutgers University, New Brunswick, New Jersey 08854

E-mail address: fluo@math.rutgers.edu

School of Mathematics and Physics, The University of Queensland, Brisbane, QLD 4072, Australia

E-mail address: tillmann@maths.uq.edu.au

Department of Mathematics, Rutgers University, New Brunswick, New Jersey 08854

E-mail address: tianyang@math.rutgers.edu 